\title{
The Influence of a Blind Professor in a Bioengineering Course
}

\author{
Benjamin R. Greenvall, Amanda L. Tiano, Anjali Chandani, \\ and Mona S. Minkara (D) \\ Department of Bioengineering, 206 ISEC, Northeastern University, 805 Columbus Avenue, Boston, MA 02120, USA
}

(Received 4 February 2021; accepted 3 May 2021; published online 1 June 2021)

\begin{abstract}
Although there is increasing literature on blind and visually impaired students in science, technology, engineering, and mathematics (STEM), there is a prevalent gap in the literature regarding STEM educators who are blind or visually impaired. This account aims to partially fill this gap by presenting the methodology and implementation of teaching by Dr. Mona Minkara, a blind bioengineering professor, as well as the tangible outcomes of this approach. We discuss the efforts taken by Dr. Minkara and a team of access assistants to develop accessible methods for teaching a largely visual course, including the use of assistive technologies, such as alternative text, braille, and text-to-speech software. Outside perspectives from teaching assistants, access assistants, and students are also discussed. Student feedback was collected in an end-of-term survey and analyzed to obtain quantitative and qualitative data. Evidenced by student feedback on their experience, we demonstrate that Dr. Minkara's visual impairment altered student perceptions about blindness in education and led to a more interactive and engaging learning environment for her students. This evidence also shows that students were overwhelmingly in support of more blind educators in STEM. We present this account and share our developing toolbox to demonstrate that a career in higher education can (and should) be accessible if given the right modifications. Efforts aimed at broadening the participation of blind and visually impaired individuals in STEM education can continue to alter student perceptions and lead to enhanced learning environments, as well as encourage instructors to increase the accessibility of their own teaching.
\end{abstract}

Keywords-Higher education, Underrepresentation, Inclusivity, Instructional design, Student perception.

\footnotetext{
Address correspondence to Mona S. Minkara, Department of Bioengineering, 206 ISEC, Northeastern University, 805 Columbus Avenue, Boston, MA 02120, USA. Electronic mail: m.minkara@northeastern.edu
}

\section{INTRODUCTION}

A 2017 study published by Bourne et al. estimated that globally in 2015, 36.0 million people were blind, and 216.6 million people suffered from a mild to severe visual impairment. ${ }^{1}$ The World Health Organization estimates the global total to be significantly higher, with 2.2 billion people visually impaired or blind. ${ }^{2}$ In the United States alone, an estimated $2.3 \%$ of the population (7.44 million) reported a visual disability in 2017. ${ }^{3}$ In spite of the improvements in rights and accommodations for persons with disabilities in the US, such as The Americans with Disabilities Act and its amendments, the education and employment rates of visually impaired persons remains limited. Of working-age people (21-64 years) with a visual impairment, only $15.9 \%$ earned a Bachelor's degree or higher, $44.2 \%$ were employed, and $30.5 \%$ were employed full-time, compared to $34.5 \%, 79.4 \%$, and $60.3 \%$, respectively, for persons without any disability. ${ }^{3}$ Those who are employed are most likely to work in retail, medicine, manufacturing, or entertainment, most commonly reporting positions as janitors, cashiers, health assistants, and retail salespersons. ${ }^{4}$ As such, the number of people with a visual impairment employed as educators is expected to be limited, and even more so in higher education. Indeed, the 2017 American Community Survey estimated that 1.68\% $(\sim 33,000)$ of workers with a visual difficulty were elementary or middle school teachers, while only $0.67 \%(\sim 13,000)$ were post-secondary teachers. ${ }^{5}$ However, the data is somewhat limited in scope, as it lacks detail on their employment in various types of post-secondary institutions, including junior colleges, community colleges, professional schools, and public 
and private colleges and universities. Comparatively, the American Printing House for the Blind found that there were 63,501 students in K-12 educational settings that met the federal definition of blindness in $2018 .^{6}$ Although specific data is not known, given the prevalence of visual impairment in the US $(\sim 2.3 \%)$ and the total undergraduate enrollment in 2018 (16.6 million) provided by the National Center for Education Statistics, ${ }^{7}$ we can speculate on the numbers of students with a visual impairment in higher education. Thus, these data suggest the number of students with a visual disability is higher than the number of educators with a visual disability. As one might suspect of the relevant literature, the majority of publications focus on the student, ${ }^{8-21}$ and there are few works focused on the visually impaired in the role of educator. ${ }^{22,23}$

Due to these limited resources and lacking sense of community, the transition to a faculty appointment can be challenging for those with a visual impairment. The PI of this work, Dr. Mona Minkara, is blind, having lost her vision at a young age. In a previous publication, Dr. Minkara reflected on the toolkit she built in order to succeed in her doctoral studies in Chemistry. ${ }^{15}$ Although she was-after some initial apprehension from her department - a Teaching Assistant (TA) for General Chemistry during her third year, the previous publication focused on her role as a student and learner. Upon completion of her Ph.D. and subsequent post-doc, Dr. Minkara transitioned to a faculty role. This role brought the additional challenge of a discipline transition from Chemistry to Bioengineering, which required her to learn new material in order to teach a course, Biomolecular Dynamics and Control (BioE 3380 at Northeastern University), which focuses on applying the principles of chemical kinetics to biology.

In addition to being one of a small community of blind scientists, ${ }^{24}$ Dr. Minkara is one of a limited number of blind or visually impaired faculty and, to the best of our knowledge, she is the only blind Bioengineering Professor. While blind instructors have a rich history as educators, ${ }^{25-27}$ we were unable to find a substantial amount literature about the specific mechanics of developing and running a STEM course at the undergraduate level. While this paper does not address the causes responsible for these limited numbers, we have observed both societal and educational impacts from students taking a course with a blind professor. For example, in an end-of-semester survey, one student commented that his parents were "initially mad" to learn that his professor was blind, but this perception changed over the course of the semester (Table S7, Student 34). We believe it is imperative to share these experiences to demonstrate the impacts, change perceptions, and assist in broadening the par- ticipation of blind and visually impaired individuals in higher education.

In this paper, we will discuss the methodology and implementation of teaching, along with efforts to improve accessibility, and the tangible outcomes of this approach. This includes efforts both inside and outside of the classroom such as the role of alternative text, screen readers, braille, lecturing, and student participation. We will also discuss outside perspectives from Dr. Minkara's access assistants ${ }^{28}$ and TAs, as well as feedback from students. To create a learning environment that was accessible to herself, implementation of Universal Design for Learning (UDL) techniques lead to a classroom that was accessible to all. ${ }^{29-31} \mathrm{We}$ will demonstrate that Dr. Minkara's visual impairment altered perceptions about blind individuals, increased awareness about accessibility, and, ultimately, led to a more active learning environment. ${ }^{32-35}$

\section{PRIOR CONCERNS}

Dr. Minkara joined the Northeastern Bioengineering faculty in August 2019 and would begin teaching Biomolecular Dynamics and Control in January 2020. This course draws heavily on chemical kinetics, of which Dr. Minkara is familiar, but the course takes an engineering approach to apply chemical kinetics to biological systems. Therefore, she first needed to become familiar with the material in order to teach the course. More dauntingly, Dr. Minkara would have to find a viable way to prepare for each class and present the course material. Unlike her sighted peers, Dr. Minkara would not have the luxury of being able to read from a set of notes during lecture. Because each class period would last an hour and forty minutes, there would be a large amount of material to convey, which again would require a detailed approach - an approach that Dr. Minkara had not yet developed.

Teaching a new class would require a tremendous amount of time even for a sighted person, but for Dr. Minkara, the task was more challenging due to her blindness. For example, braille notes could provide both a means to memorize the lecture content and recall information during lecture. However, Dr. Minkara does not read braille at a sufficient level to be able to quickly read information. In theory, it could also be possible for Dr. Minkara to receive audio prompts during lecture using a headset and text-to-speech software. In reality, when in testing this setup, we found it did not work well due to the difficulty in speaking aloud while listening, which interrupted the flow of the lecture. Still, Dr. Minkara would need to convey the visual content and mathematical details of the course when she would not be able to see them 
herself. Considering the possibilities, the most feasible - albeit, time-consuming-option was memorization.

\section{CLASS PREPARATION}

\section{Creation of Materials}

In order to familiarize herself with the material, Dr. Minkara attended a section of the same course, BioE 3380, during the Fall 2019 semester. Attending this course formed a strong basis for the material that Dr. Minkara would present in her own class. However, the delivery of the material, writing out the notes by hand, was not something Dr. Minkara could replicate. Instead, Dr. Minkara decided to provide an outline of the lecture to the students, which they could fill in as she went through the lecture. ${ }^{36-38}$

During the prior semester, Dr. Minkara created a digital document for each lecture that she would give with the help of a TA. This required derivations to be typed and figures be digitally created. For each equation and figure, descriptive alternative text was added to verbally describe the figures and equations. Then, Dr. Minkara would determine the key information, such as definitions, equations, and examples, that needed to be removed to create a 'fill-in-the-blank' copy for students, generating a framework on which they could take notes. This lengthy process would not have been possible without the support of her TA and the Bioengineering Department, which provided a TA to support her preparation even though she was not teaching that semester. Recalling the process, the TA remarked: "When I first started working with Mona, she had high expectations for my quality of work. [...] In my experience with Mona, I found that this approach helped me produce work I was proud of and made for a productive semester, as well as helped in my development as a graduate student in my first semester." In addition to being a unique and enriching experience for the TA, this was an invaluable accommodation that largely contributed to Dr. Minkara's success.

\section{Memorization}

With much of the physical material prepared in the Fall semester, Dr. Minkara was able to focus her time during the Spring semester on preparing to present the material. In the week leading up to each lecture, Dr. Minkara and her access assistant, Ben Greenvall, would spend several hours working through the material that she would present. When time allowed, Dr. Minkara preferred to write out the lecture line by line as Greenvall read it aloud. While she was unable to see what she had written, the act of slowly writing each step helped to commit the material to memory. While this technique was effective to learn the material, this process was incredibly time consuming, and, as the semester became busier, the preparation time became mainly focused on reviewing the most challenging sections, which were often the mathematical derivations.

In a slightly more sustainable (albeit less effective) process, Greenvall would read the material aloud while Dr. Minkara listened intently, committing the material to memory. During this process, they would pause to discuss each step of the mathematical derivations and every plot and figure. During these processes, it was necessary that the material be read clearly and correctly, especially when working through a mathematical derivation, as any misconceptions would persist until corrected. Her access assistant, Ben, recalled the process: "Because reading aloud was an intermediate for Dr. Minkara to engage with the material, our process inherently had more opportunity to introduce an error than if someone were just reading and silently memorizing the material themselves. As a result, we were motivated to optimize the recitation process in order to minimize the potential for error and subsequent delay. We both found it helpful to develop a consistent set of terminology suited for our material. As many of the equations featured lengthy terms in quotient and exponential form, a key term in our discussions was to use 'baseline' to denote returning to the 'ground' state of the equation. So, by using the phrases 'raised to', 'divided by', and 'baseline', it would typically be clear which part of the equation we were currently working with."

It is important to note that when reading materials, it is also helpful to consider the end-user and their background. We found it particularly useful when reading and describing mathematical equations to incorporate Dr. Minkara's chemistry background. For example, chemists understand quantities written in brackets, e.g. $[\mathrm{A}]$, to represent molar concentrations, and the presence of the subscript ' 0 ', e.g. $\left[\mathrm{A}_{0}\right]$, represents an initial concentration at the start of a reaction. This knowledge can help the access assistant reading the material to present the information in a way which is clearer to the end-user. Other end-users are likely to have different expertise and preferences; therefore, it is critical to have a discussion of what works best for them.

When preparing for a lecture, Dr. Minkara would focus on memorizing the exact flow of the lecture, paying particular attention to the order of each mathematical step so as not to confuse the students following along during lecture. She felt that it was 
essential that her recitation match exactly what was on the screen. However, her preparation did not focus solely on memorizing the content of each document. During the memorization process, she spent a considerable amount of time preemptively considering the potential questions students might ask. This preparation was particularly relevant to the mathematical and theoretical content, as there are often several different ways to solve a problem or understand a concept. Therefore, while it was vital that Dr. Minkara's memory matched the approach that she had used in the prepared lecture material, it was also important to consider the different backgrounds and perspectives that her students might bring. This approach both helped her to be prepared for students' questions, and ultimately, made her a better lecturer, as she was able to frame the potentially confusing parts of the lecture with better clarity.

Though complete memorization was effective, it was extremely time intensive, and we explored ways to make lecture preparation more efficient for Dr. Minkara. In the next two sections, we will discuss the use of alternative text with VoiceOver, the Apple screen reader software, and a braille notetaker.

\section{Alternative Text}

The initial creation of the lecture material was not readily accessible to Dr. Minkara and therefore did not allow her to review the material and memorize it on her own. Studies have shown that universal design is effective for all persons, ${ }^{29,30,39,40}$ not just those with disabilities, therefore, instead of solely focusing on creating accessible documents to Dr. Minkara, our aim was to work towards creating universally designed content that would be accessible to both Dr. Minkara and her students. Our first task was to make images, graphs, and figures accessible by adding descriptive alternative text, commonly referred to as alt text, throughout. ${ }^{41,42}$ Upon completing this task, we found that there were persistent accessibility issues (see Appendix A, Example 1). Some portions of the written material would read correctly, but other areas could not be detected by the screen reader, particularly autoformatting (bullets and numbering), figures, groups of objects, and equations. The issue was likely compatibility between Microsoft Word, which was used to create the lectures, and VoiceOver on Mac, which was used for screen reading. We turned off autoformatting and all bullets and numbering were replaced by a special dot character, • (Unicode character 2022), or plain text for lists, A., B., C., in order to maintain the aesthetics and readability of the documents for students, while making it compatible with VoiceOver.
A predominant obstacle with the lecture material was the large quantity of equations and mathematical symbols present, which could not be read by VoiceOver. There are a variety of tools available for improving the accessibility of mathematical equations through screen readers and/or braille displays such as MathML (https://www.w3.org/Math/), MathJax (htt ps://www.mathjax.org/), and MathType (http://www. wiris.com/mathtype), but these tools help primarily with web-based content. For ease and compatibility, we decided to use the alt text functions within Microsoft and quickly found that adding alt text for equations is possible in PowerPoint, but not in Word (Appendix A, Example 2). In order to circumvent this issue, each equation was saved as an image, subsequently inserted back into the lecture document, and then alternative text was added.

This process of adding alternative text was particularly frustrating due to the number of unforseen obstacles encountered, as Dr. Minkara's access assistant, Dr. Amanda Tiano, recalls: "Initially, I was very confident in my ability to make the lecture materials accessible having created ADA-compliant documents for undergraduate-level chemistry courses without any known issues in my previous position. However, it turned out that this problem was more challenging and complex than my initial expectations. Reflecting on my previous experience, I think the key word is 'known'. To the best of my knowledge, none of the students in those courses had a visual impairment or required the use of speech-to-text software, and now I wonder whether those documents were truly accessible, as the accessibility checkers in Microsoft Office and Canvas led me to believe. In this case, however, we received instant feedback on our efforts from a visually impaired user which was informative, invaluable, and, at times, frustrating. Indeed, the compatibility issues we encountered between Office (used to generate the document) and VoiceOver (output) was vexing when the accessibility checker did not identify any issues, but the feedback Dr. Minkara provided enabled us to systematically identify specific problems and work towards solutions. Overall, this experience was illuminating and increased my awareness of accessibility when creating documents."

The last major difficulty with this approach was the nature of the equations in the lecture material. There are numerous, intricate equations throughout, which can make alternative text difficult. The access assistant working on the alternative text should have an understanding of both basic and advanced mathematical concepts, e.g. power, integrals, linear equations, matrices, etc., in order to correctly describe the content. The access assistant must also be attentive to 
detail to ensure also that the alternative text is clear and accurate. As an example, consider Eq. [1],

$$
k=A e^{\frac{-E_{a}}{R T}}
$$

which depicts the Arrhenius Equation. This equation is not inherently complex but consider the way in which an access assistant may type a description of the equation. If the access assistant is familiar with the equation and assumes the end-user is as well, he or she may transcribe it as " $\mathrm{k}$ equals $\mathrm{A}$ e to the negative $\mathrm{E}$ a over RT". However, if the end-user is not already familiar with this equation, it might not be clear that the first A and e are multiplied and that the second 'a' is a subscript. Additionally, the word 'over' is commonly used to indicate a division, but it is not clear from the description where the division occurs. Lastly, there is no distinction between uppercase and lowercase letters, so the user may not be able to identify a difference between ' $A$ ' and ' $a$ ' and ' $E$ ' and ' $e$ ' in the above description. Using VoiceOver, uppercase and lowercase letters can be distinguished with a different pitch, but the user must scroll character by character to obtain this information. Although possible, it is timeconsuming, and the end-user would need to be made aware that different cases are present. Considering all the potential sources of error, there are at least 66 different equations that could be understood from this combination of terms. It is highly likely to result in an end-user who is misinformed, and this incorrect information could then be shared with the students, if not corrected.

The previous example is a simple one. Now consider Eq. [2],

$$
[A]=\frac{\left(\left[B_{0}\right]-\left[A_{0}\right]\right) \frac{\left[A_{0}\right]}{\left[B_{0}\right]} e^{-k t\left(\left[B_{0}\right]-\left[A_{0}\right]\right)}}{1-\frac{\left[A_{0}\right]}{\left[B_{0}\right]} e^{-k t\left(\left[B_{0}\right]-\left[A_{0}\right]\right)}}
$$

which describes the concentration of a species in a second order reaction. It is evident that Eq. [2] is much more complex than Eq. [1] and, therefore, the potential for mistakes and misinformation is much greater. It is critical for access assistants to use more descriptive words such as "the product of", "the quotient of", and "to the power of" and indicate the presence of parentheses to ensure the end-user can identify blocks of information more clearly. Additionally, for equations including quotients, it is important to indicate when an equation is brought back to 'ground state'; in our case the word 'baseline' was used to indicate this. Taking into account these guidelines, two examples of accurate alternative text descriptions of Eq. [1] are: (1) $\mathrm{k}$ is equal to capital A times e raised to the quantity of negative capital e subscript a divided by $\mathrm{R}$ times $\mathrm{T}$ and (2) $\mathrm{k}$ is equal to capital A times e raised to the power of open parentheses negative capital E subscript a divided by $\mathrm{R}$ times $\mathrm{T}$ end parentheses. An alternative text description for Eq. [2] added through Microsoft PowerPoint is included in Appendix A, Example 3.

Having established a clear method to create accessible documents, we documented the step-by-step process to enable any access assistant or work-study student to work on the remainder of the lecture material. The final step in the process was establishing a library of equation images and their corresponding alternative text. This will enable us to generate new lecture material in the future more rapidly while maintaining accessibility. Although established with teaching in mind, these methods and tools can also be utilized for Dr. Minkara's research, which will assist her research students in preparing accessible manuscripts, theses, digital lab notebooks, and presentations. Although Dr. Minkara was our only user of the alternative text, we felt it was important to keep universal design in mind and prepare for the possibility of a blind or visually impaired student in her classroom. Therefore, another blind or visually impaired individual using our documents should find them to be fully accessible, regardless of their previous knowledge or background. By sharing our process, we aim to provide methods and tools to create sustainable systems that can be proactively applied by anyone to make education accessible to everyone. These principles can also be extended beyond the classroom, e.g. publishers ensuring that journal articles are accessible with alternative text.

\section{Braille Notetaker}

Six weeks into the semester, we also investigated the use of a braille notetaker to improve accessibility. Interestingly, in the United States less than $10 \%$ of blind and visually impaired individuals are braille readers. ${ }^{43}$ In spite of having lost her vision at a young age, Dr. Minkara did not learn braille until very recently. The aim was to use the notetaker to generate a high-level outline of the lecture materials that could be used by Dr. Minkara while preparing for class and during lecture as a quick reference. Additionally, she could use the device to review the now accessible course documents without an access assistant. We note that tactile readers in the blind and visually impaired community may not consider these materials accessible, as text is used to describe the equations, rather than Nemeth braille. However, these documents are fully accessible to Dr. Minkara using her current tools and existing knowledge of braille.

We used a BrailleSense Polaris (HIMS, Inc.) which has a 32-cell braille output, a Perkins-style keyboard, and comes equipped with a variety of accessible soft- 
ware including Google and Polaris's own office apps for email, documents, presentations, and spreadsheets. ${ }^{44}$ The notetaker can also be connected to an external display or used with the application TeamViewer to share material with a sighted access assistant, which can help with navigation. Once set up, an access assistant can review the lecture content while Dr. Minkara types up basic notes and/or an outline for reference. Using this technique, Dr. Minkara can have a set of notes that can be quickly referenced. Additionally, using USB or Wi-Fi, the full lecture materials can also be reviewed with the notetaker. This allowed Dr. Minkara to prepare for upcoming lectures at her own convenience, especially when her access assistants were not available.

Based on Dr. Minkara's's previous experience with the braille notetaker, we believe that there is much more that can be accomplished with the device for teaching, but additional training is needed to expand its usage. Unfortunately, further use of the braille notetaker was impeded by the COVID-19 pandemic, which moved classes and non-essential work from inperson to online. Thus, Dr. Minkara was unable to work with her access assistants to explore additional uses.

\section{CLASS EXPECTATIONS}

On the first day of Biomolecular Dynamics and Control, Dr. Minkara addressed the class and told them that the course would be unlike others they had taken in the past. For the students to get the most out of their time together, they would need to engage with both her and their peers to a much greater degree than other science classes. To promote engagement and maintain a direct line of contact between herself and the students, Dr. Minkara made the following requests of the students: (1) ask any questions they may have aloud instead of raising their hand, (2) at least initially, preface their question with their name, (3) respond audibly to questions, and (4) volunteer for demonstrations, board work, and reading material aloud. These requests would allow Dr. Minkara to (1) field questions directly, (2) match names to voices, (3) gauge the classes' comprehension and speed of instruction, and (4) ensure class progression and success, respectively. The goal of these requests was to encourage the students to meet Dr. Minkara at the level where she felt that she - and they-could be most successful. Although these requests were not necessary, asking students to make these accommodations made her a better educator. The end result was a dynamic, active learning environment, which was atypical for such a mathematically intensive, mid-sized, 'lecture-style' course.

While working through the lecture document, which the students received in a 'fill-in-the-blank' format, Dr. Minkara's approach to instruction involved frequently introducing opportunities for discussion, question, or activity. Students were often asked to participate in a variety of ways. For instance, students were asked if they had any familiarity with a new term, example, or mathematical approach before the completed section of the presentation was revealed, which gave students the opportunity to draw upon prior knowledge. Students were also asked to read the material directly from the completed notes to create a change of pace and prompt students to take a more active role in lecturing and teaching their peers. Lastly, students were asked to work collaboratively in both small groups to answer questions or together as a class to solve problems at the board. In the latter case, Dr. Minkara applied a condition that the student at the board was to only write what the class told them, which removed the pressure associated with thinking on the spot.

While participation in this way was initially met with hesitation, Dr. Minkara maintained her rules of engagement. Though awkward stretches of silence occurred in the first few days, Dr. Minkara would stand in front of the class and wait until she got an answer to her question. There were also instances in which student tried to interact with her sighted access assistant instead. Greenvall, Dr. Minkara's access assistant remarked on his first-hand observations, "Initially, there were times where students would raise their hands during lecture and make eye contact with me, expecting that I would tell Dr. Minkara. Whenever this happened, I would either direct my gaze towards Dr. Minkara, pretend not to see the student, or just shrug, to encourage the students to interact directly with her. As the semester progressed, these interactions became less frequent as the students continued to embrace Dr. Minkara's process." After a few class sessions, the students began to participate more frequently and with less reluctance, and it became clear that the system was one that many of them accepted and enjoyed. In the end of term survey, one student commented, "Yes, it was weird to answer every question instead of just nodding, it was weird to read out loud from the slides, but it got me engaged. I was present in the class, paying attention, because Mona made it interactive" (Table S2, Student 20). Another student commented: "At this point in my academic career professors feel that taking notes and participating in class is up to the student. I appreciated that Dr. Minkara pushed us to participate and be engaged as I think it helped with learning as well as general interest in the material. It 
was different in the best way" (Table S4, Student 7). Their participation became so significant that, eventually, the ease and enthusiasm with which the class responded became a proxy for the students' overall morale.

By the end of the semester, nearly every student had participated during class discussions. Students acknowledged that the classroom was a welcoming and respectful environment as one student commented, "She fosters an environment in class that makes everyone feel comfortable to ask questions and show when they are confused, which has never happened in another classroom that I have had" (Table S4, Student 33). By embracing Dr. Minkara's process and actively participating, students learned valuable communication skills while providing the essential verbal cues Dr. Minkara needed to be successful.

Outside of the classroom, Dr. Minkara held office hours twice a week. Initially, she was worried about how office hours would go, as she felt that many of the potential questions would be visual. When time allowed, she made sure to prepare well for these sessions by re-reading the material for that week and the previous week's quiz and problem sets, however this was not always possible.

When students came with a question, Dr. Minkara would ask the student to read the question aloud and describe the problem to her. Ultimately, this had two benefits; it would allow Dr. Minkara to recall the problem and familiarize herself with their point of confusion, and it forced the student to think critically about the problem and precisely articulate whatever concept was not making sense. Often, the student would answer their own question while discussing the problem, as one explained: "In some ways, Dr. Minkara being blind actually made office hours better, as in reading the problem/my work out loud, I often figured out the error in my work when I otherwise wouldn't" (Table S5, Student 28). If needed, Dr. Minkara and the student would solve the question together aloud. Because the office hours were often well attended and many of the same questions arose, Dr. Minkara would encourage students who understood the material to teach their peers with her supervision, often by working through a problem on the board. This process allowed students to further their understanding by explaining concepts to peers. This process had the added benefit that Dr. Minkara could ask the other students to check the work of the student writing on the board, though printed solutions and one of Dr. Minkara's access assistants would be on hand to serve as a visual backup if necessary. Because Dr. Minkara encouraged the students to discuss the material amongst themselves, it created collaborative, supportive connections that carried over into the classroom. In the end of term survey, the students who attended office hours indicated that they found her office hours to be effective (Appendix B, Table S5).

\section{EFFECTS OF REMOTE CLASSES}

During March 2020, the novel SARS-CoV-2 virus required quick conversion of the in-class design to a digital one. This adjustment had significant impacts on Dr. Minkara's teaching process, which required her to change both her preparation and presentation approach. Dr. Minkara was no longer able to work with her access assistants in person. It was difficult for Dr. Minkara to memorize the lecture content to the same level of detail, as the already time-consuming process of memorizing only became more challenging while working remotely. For instance, it would no longer be possible for her access assistant to review the material that Dr. Minkara had written down or create physical flash cards for her to hold. Even a relatively simple process like tracing her hand along a printed graph so that she could get a rough sense of the 'shape of the data', which could typically be completed in about a minute while in person, took up to $15 \mathrm{~min}$ to describe while working remotely.

After experimenting with various digital platforms, Dr. Minkara decided to hold class sessions and office hours through the video conferencing app, Zoom, as it was the most accessible for her to navigate remotely. Unlike the engaged and responsive class sessions that Dr. Minkara was used to having while on campus, the virtual sessions noticeably lacked connectivity due to the nature of the situation, as one student noted, "To be quite frank, the transition to online classes disappointed me the most because of this class as it was the most enjoyable I had in terms of content and environment" (Table S8, Student 13). To minimize feedback in digital lectures, the norm is for students to leave their microphones muted while not talking, but Dr. Minkara was used to using student response to gauge comprehension while in person.

To deal with these preparation and presentation challenges, Dr. Minkara significantly changed the operational structure of the class. She provided students the completed version of notes with the expectation that they would read them prior to class. Then during class time, Dr. Minkara talked through the major concepts of the material as her access assistant displayed the document via screen sharing, but she would not initially explain the material in full detail, allowing the students to ask questions about the specifics. In this new system, students would not need to spend time copying the material-instead, Dr. Minkara hoped for a discussion driven by the students' 
questions. This approach allowed Dr. Minkara to navigate the challenges of memorizing and working with her access assistants remotely, though the transition was certainly a jarring shift of pace for the students. Having observed both teaching environments, Greenvall commented, "While Dr. Minkara's in-class sessions relied heavily on student interaction and participation, the way we needed to operate online made this significantly more challenging. With nearly 40 people coming together on a video call, much of what made Dr. Minkara's approach effective (and for many students, enjoyable) wasn't possible online. It's hard to expect students to shout out a question mid-lecture in a virtual class that size, and even if they could, we wouldn't have been able to prepare the material to make a traditional lecture possible." Thankfully, the class had already spent several months together, and because Dr. Minkara had built a rapport with the students, they were understanding of the challenges she faced in maintaining an engaging class while working remotely.

Although the situation was not ideal for either Dr. Minkara or her students, the online experience speaks highly of her effectiveness while teaching in person. Many students often think that if given detailed notes, as became necessary when working virtually, they would be able to sufficiently learn the material on their own. On the contrary, in Dr. Minkara's class, several students were disappointed to lose the old system as one student commented in the university course eval-

TABLE 1. List of survey questions.

Number Question

$1 \quad$ Before starting class, what preconceived notions or expectations did you have about Professor Minkara's ability to teach as a blind professor? Please also indicate if you didn't know she was blind

$2 \quad$ How did these preconceived notions and expectations compare to your experience in the course?

3 Did it make a difference to you that such a visual course was taught to you by Professor Minkara as opposed to by a sighted professor?

$4 \quad$ Did it make a difference to you that such a visual course was taught to you by Professor Minkara as opposed to by a sighted professor? Please explain

$5 \quad$ Did you find that Professor Minkara's teaching methods differ from other professors?

$6 \quad$ Did you find that Professor Minkara's teaching methods differ from other professors? Please explain

$7 \quad$ Did you find Professor Minkara's office hours to be effective?

$8 \quad$ Did you find Professor Minkara's office hours to be effective? Please explain

9 If you found yourself in a teaching or mentoring position in the future, do you believe you would be able to effectively teach or mentor a blind student?

10 If you found yourself in a teaching or mentoring position in the future, do you believe you would be able to effectively teach or mentor a blind student? Please explain

11 Reflecting on your experiences in this course, do you think there should be more blind educators in STEM?

12 Reflecting on your experiences in this course, do you think there should be more blind educators in STEM? Please explain

13 Please provide any additional comments that you would like to share regarding Professor Minkara's teaching as a blind instructor

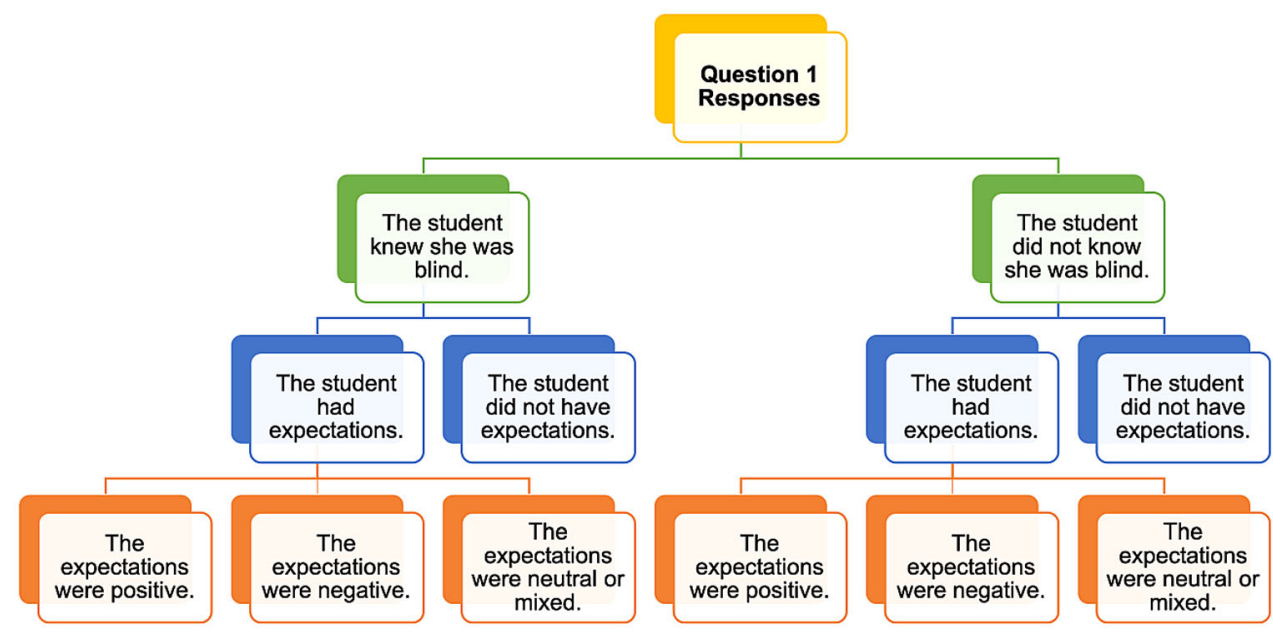

FIGURE 1. Example of breaking down possible outcomes for Question 1. 




FIGURE 2. Survey responses comparing students' initial expectations (Question 1) to their experience (Question 2). Responses are grouped by students who knew (solid, bottom) and did not know (patterned, top) that Dr. Minkara was blind prior to the course and colored green, red, yellow, and grey for positive, negative, neutral, and none, respectively.

uations "Prof. Minkara is a fantastic lecturer. When the class transferred to online, I found it difficult to learn the material without the lectures." Transitioning to a virtual format further affirms much of what we have observed throughout this process; there are educational and experiential benefits to the way Dr. Minkara conducted her class while in person, but the approach required a lot of time, coordination, and effort. Together, these provide evidence that it is vital to continue developing efficient, sustainable, and accessible systems for Dr. Minkara to teach, especially with the state of higher education constantly evolving.

\section{STUDENT FEEDBACK}

Towards the end of the semester, we developed a 13question survey to obtain feedback from students on their experience Dr. Minkara's class. The general format of the survey was a yes/no question and a secondary question prompting students to explain their previous response. The full list of questions is shown below in Table 1.

The survey was available electronically to students via Blackboard, in order to track completions while keeping responses anonymous. Students received extra credit for completing the survey.

\section{Analysis}

Of the 36 students in the class, 34 students (94\%) completed the survey. For some questions, we used a tree diagram approach to first determine all possible outcomes (Figure 1). Next, each student response was reviewed and subsequently binned to one of the outcomes. It is important to note that the binning process is inherently more subjective than objective, in particular for the neutral or mixed responses, which contained both positive and negative attributes. However, we had multiple reviewers and collectively discussed the responses that were difficult to assign to minimize subjectivity. Using this approach, we were able to obtain both qualitative and quantitative data from the questions with open-ended responses.

The full set of survey responses is available in Appendix B in Tables S1 through S8. Some student responses incorporated into the text have been altered for clarity or typographical errors and are indicated as such with brackets. Each quote is followed by a table number and a number between 1 and 34 for reference to the original, unedited response in each table. We also note that this research was determined by Northeastern University's IRB as exempt from classification as research involving human subjects.

\section{Questions 1 and 2: Impacts and Perceptions}

The first two questions focused on understanding the societal impacts and perceptions of the students on having a blind professor. We began by asking students whether they were aware of Dr. Minkara's blindness before class began and what preconceived notions or expectations they had regarding her blindness (Question 1, Table S1). Nearly $60 \%$ of the class (20 of 34 students) knew of her blindness prior to class. Of the 20 students that knew, 15 had some preconceived no- 
tions or expectations related to her blindness, the majority being positive ( 9 students), and the remainder split between negative and neutral (Figure 2). Several students expressed that they enrolled out of genuine curiosity in what it would be like taking a class with a blind professor, while others cited making a collective decision with their peers and their trust in the university to hire qualified faculty. One student enrolled because "to have the drive to teach a class in the engineering field, blind, [the professor] would have to really be passionate about the material and teaching as a whole" (Table S1, Student 2). Interestingly, a few students stated that it was part of their decision to take Dr. Minkara's section of the course, "My perceived notion was that I would be deeply inspired and pushed out of my comfort zone in terms of the learning style" (Table S1, Student 1).

Among the students who conveyed neutral (mixed) or negative expectations, some students identified themselves as visual learners, and were worried they may not be able to learn from Dr. Minkara. Students were apprehensive about learning material with visual aspects, such as graphs, images, and equations, from someone lacking the physical ability to see them. One student focused on the mathematics, "As a visual learner, I was concerned about how I would be able to learn complicated math problems" (Table S1, Student 22). Overall, whether students had expectations or not, a common theme was that many students believed that the course would be different and wondered whether Dr. Minkara's blindness would affect, for better or for worse, the class structure and, ultimately, their learning.

Next, we asked the students to compare their initial expectations or preconceived notions surrounding Dr. Minkara's blindness to their experience in the course (Question 2, Table S2). Regardless of whether the student had positive, negative, neutral, or no expectations, the majority of students $(91 \%)$ reported a positive experience, while only $9 \%$ ( 3 out of 34 ) expressed a neutral (or mixed) experience in their response (Figure 2). Students expressed that the experience surpassed their initial expectations, "My expectations were not met at all, they were exceeded. I loved how the class was taught and how energetic the environment was. I thought that the class would be a lot of videos and some lectures, but the format of the class definitely took me by surprise" (Table S2, Student 16). Several students who initially had negative or neutral expectations also conveyed that they were incorrect in their expectations, "Walking in the first day and finding out that Dr. Minkara was blind I was a little nervous about the class. These notions were SO WRONG. [...] I had an incredible experience in this course" (Table S2, Student 7). Considering the student responses for Questions 1 and 2, it is evident that because of their experience in Dr. Minkara's course, students' perception of blind or visually impaired individuals in education was changed in a positive way. We anticipate that over the course of Dr. Minkara's career, she will continue to alter perceptions of the blind and visually impaired among students and faculty, which will lead to positive societal impacts such as increasing the number of blind and visually impaired persons in higher education.

\section{Questions 3 Through 6: Differences in Having a Blind Professor}

Students were also asked whether they felt there was a difference in being taught by Dr. Minkara as opposed to a sighted professor, and to elaborate on their response (Questions 3 and 4, Table S3). The majority of students (26 out of 34) responded that it did not make a difference that Dr. Minkara was blind. Overall, many of these students discovered that vision was not necessary to make a great professor; they emphasized that skills, abilities, and teaching methods were what led to their overwhelmingly positive experience in the course, "I don't think that ability to teach depends on the eyesight. It depends on ability to find an approach, to be clear, to have passion for a subject one is teaching and to be passionate about teaching in general" (Table S3, Student 20). Another student provided their unique perspective on their experience, "It did make a difference for me. As a deaf student, visuals are something that I rely on heavily. This class made a difference in a positive way because I felt that the class was perfect for someone with my impairment as it was taught by someone who is also disabled. Being able to meet someone with a more impactful disability than mine was also a great learning experience for me as I have seldom met other deaf people and have never met a blind person before" (Table S3, Student 29). This response is particularly valuable and adds an additional element to the impact of Dr. Minkara's teaching. Given that deaf persons rely on visuals while blind persons rely on auditory input, it's easy to assume that it would be impossible for a blind person to teach a deaf person. However, it was shown that it is not impossible, but rather, that it was a positive experience for the deaf student. Overall, these responses provide clear evidence of the larger impacts of Dr. Minkara's blindness in education, not only as a role model for other disabled persons, but also in teaching students that sight is not a requirement for a great educator.

However, not all of the students felt the differences were positive. One student expressed that they had difficulty with Dr. Minkara's approach, "A lot of professors [nowadays] lecture by writing the notes out 
with the class. Personally I find that helpful and have learned to expect that in most of my classes so it was difficult for me [to] adapt to Professor Minkara's teaching style. The packets were helpful in that it gave us a visual guideline to the lectures however it was one of those instances where it made it more difficult to know when I didn't understand a topic because I was just focused on filling in the blanks not understanding what I was writing. I think students also tended to just try to learn the material they didn't understand themselves or with each other rather than in the classroom where it is easier to just ask a sighted professor to further write out calculations or explain a visual concept more on the spot" (Table S3, Student 22). Although the responses were largely positive, some students still have reservations about visual aspects of the course and, therefore, more work is still needed to assuage doubts. By continuing to engage with students both inside and outside the classroom, we hope to minimize and, eventually, eliminate these notions by demonstrating that it can be taught effectively by someone who is blind.

In a follow-up question, students were asked specifically whether Dr. Minkara's teaching methods were different from other professors and to explain their answer (Questions 5 and 6, Table S4). Of the responses, $88 \%$ (30 out of 34 ) indicated there was a difference, compared to $12 \%$ (4 out of 34 ) who stated there was no difference.

Two of the major differences specified by students were the blanked lecture notes and degree of participation. Although some students expressed difficulty adapting to Dr. Minkara's methods, students largely enjoyed the blanked lecture notes provided for each class, "this method actually made the class more effective. Since we had all the essential notes we needed on paper, we could focus more on questions" (Table S4, Student 16). Coupled with the participation required, many students expressed that her methods were effective and engaging. "Professor Minkara demands participation in her course. She wants her students to be present in class and makes an effort to make sure they all understand the material. She consistently asks the class for feedback. This is more interaction than the rest of my professors combined" (Table S4, Student 4). Nearly all student responses depicted some aspect in which the course differed.

Overall, the responses to these questions demonstrate that having a blind professor is different for students, largely in a positive way. We believe this shows that as a result of Dr. Minkara's methods students identified teaching methods that differed from other professors, methods which led students to obtain a better understanding of the material and also taught students how to communicate more effectively.

\section{Questions 9 through 12: Propagating Change}

Drawing on their experience with Dr. Minkara, Questions 9 through 12 of the survey prompted students to consider propagating change. First, students were asked whether they felt they could effectively mentor or teach a blind student (Questions 9 and 10, Table S6). The responses were roughly split down the middle with $53 \%$ responding positively (18 out of 34 ) and $47 \%$ responding negatively (16 out of 34 ). Of the negative responses, some students stated that they felt they needed additional training and practice to be successful, although many expressed increased awareness, "I would need additional support and training before being confident in my ability to mentor or teach a blind person. After being in Professor Minkara's class, I feel that I am more aware of the unique experiences that blind students and teachers have, but I feel I would have to adapt my teaching style to fully be able to communicate new learning material without the use of vision" (Table S6, Student 19). While others reiterated their reliance on visuals, "I find that when something is hard to explain, I instinctively reach for a pen and paper. [...] It would be difficult for me to explain a topic if I didn't have access to these tools" (Table S6, Student 32). Most students expressed an increased awareness of accessibility and an appreciation for the tools and communication skills they learned in this course. For example, "As much as I want to check yes, I believe there is so much I need to learn about how blind individuals perceive and learn so I would be better able to explain concepts and reach them. I want to teach in the future, and I desire for my classes to be as accessible as possible. Being taught by Professor Minkara gave me some insight into this" (Table S6, Student 14). Overall, the student responses are interesting given that Dr. Minkara did not explain how to teach students with disabilities and students drew upon their own experiences in the class. Asking this question prompted students to think about a possibility they might not have considered. We found that a result of their experiences in Dr. Minkara's class, many students are more willing to adapt in order to be more inclusive to persons with disabilities.

Lastly, we asked students whether there should be more blind educators in STEM (Questions 11 and 12, Table S7). The vast majority of the students answered yes (31 out of 34) along with two negative responses and one unanswered. Many students commented that Dr. Minkara's visual impairment had no effect on her ability to teach, and in some cases, students felt that this improved the course, "I mean, there's nothing blindness takes away from a professor's ability to teach that can't be fixed with a little hand-waving and a trusty TA with a computer. If anything, having a 
disability improves the class because it really forces the professor to be aware of their teaching styles. It forces them to care, and that's more than I can say of some of my able-sighted seeing professors. The effort and care a professor puts into teaching a student is what makes a professor good. I suppose this line of reasoning could be applied to other disabilities as well" (Table S7, Student 3). Other students were more emphatic in their responses, highlighting the importance of creativity and different perspectives. As one student remarked, "There should most definitely be more blind educators in STEM. To me, STEM is all about creativity. It is about approaching a problem from as many different angles as possible. It is about thinking outside the box. Blind educators bring a unique perspective to the table. They may use alternative methods to understand or explain something, which could be very useful to students" (Table S7, Student 4). Other students used Dr. Minkara as an example to broaden participation more generally in STEM, "I do not think blindness is a barrier that is too large for STEM. I am a deaf student in STEM and can speak from first hand experience that being disabled does not mean [debilitated]. Professor Minkara is an excellent example of this as she is one of the most intelligent and motivating people that I have encountered at Northeastern (and in general)" (Table S7, Student 29). The two negative responses acknowledged the possibility, but one student did not feel it would be suitable for all courses, "I don't think all courses cater well to not being visually supplemented. I don't think blind professors shouldn't be allowed, but I also don't think they should be sought after" (Table S7, Student 26). The other response felt uncertain over the use of the word 'should' in the context of blind educators, "I don't know if I would say there SHOULD be. I think there can be and it's possible, but not like they are needed. I think the word "should" makes that a difficult question" (Table S7, Student 22). The last student did not choose yes or no but felt that blindness should not be the defining characteristic, as they explained, "After taking this course, I don't think that blindness is the characteristic I would emphasize when talking to someone about prof Minkara. What I would emphasize is her charisma, her passion to teach and learn, her energy in class and her desire to make everyone feel welcomed. These are the characteristics that I think define prof. Minkara and that I think every professor should have. My guess is that part of why prof. Minkara is so great is that she decided to not let her disability to change her life and dictate what she could do. So I think blindness helped develop all of those characteristics. So, to answer the above question, I would say there should be more educators like Prof. Minkara, not necessarily blind" (Table S7, Student 16). This sentiment was also shared among many of the positive responses.

\section{In Summary}

Overall, the survey results demonstrate that students generally had a positive experience in Dr. Minkara's course, reported being more aware of accessibility issues, and were supportive of more blind educators in STEM. Although many students had to adapt to her teaching methods, the majority of students appreciated her approach and felt more engaged than they had in other courses due to the participation required for a class that normally would not require it. However, this approach did not work well for all students. A few introverted students commented that they had difficulty in participating. Thus, we acknowledge that there are drawbacks to Dr. Minkara's approach, however, the reality is that Dr. Minkara's classes have an inherent need to be more verbal. Using this valuable feedback, we hope to develop solutions to enhance student engagement and improve student experience in future courses.

\section{NEXT STEPS}

In the future, we aim to explore these tools that aid in reading equations using speech-to-text software to prepare accessible course documents. For example, iBooks Author combined with MathType or the Daum Equation Editor has shown promise in creating documents with accessible equations and text. We will also explore Job Access With Speech (JAWS), a Windowsbased screen reader software in order to compare Mac and Windows readers and the various math editor tools available. We anticipate this will enable us to streamline our methods to create accessible documents for inside the classroom and beyond.

We aspire to further our exploration with the braille notetaker to improve the lecture preparation process. With the recent availability of new tactile-display tools, such as the Graphiti by Orbit Research, graphical and visual information is becoming more accessible to the blind and visually impaired. These types of tools will enable Dr. Minkara to rely less on access assistants for verbal descriptions and become more independent in preparing for lectures in future.

These are just a few examples of improvements that will make teaching more accessible. We envision that these new technologies will also increase the prevalence of blind and visually impaired individuals in higher education. 


\section{CONCLUSIONS}

In this work, we have demonstrated that students learning from Dr. Minkara, who is blind, were positively impacted through their experience in her Biomolecular Dynamics and Control course. Although some students were initially apprehensive, particularly related to visual aspects of both learning and the course material, students largely demonstrated openness and willingly adapted to a different teaching method. As a result of Dr. Minkara adapting her approach to make teaching more accessible, the learning environment was more interactive, and students felt they were more engaged and active in their own learning. Many students also voiced sentiments of being inspired as a result of this learning experience, and overwhelmingly supported the idea of more blind educators in STEM.

This process has also been a learning opportunity for us in a number of ways. Just as there was not a textbook for the class, there was not a 'textbook' for how to develop and teach a course as a blind person. We have begun to develop a toolbox for course development and lecturing as a blind professor. Although several improvement processes are still ongoing, we aim to continue to develop our toolbox to make a learning experience that is not just accessible for one, but accessible for all. We hope that the students have expanded their toolboxes too, both in their knowledge of biomolecular dynamics and how to learn from and appreciate people with different perspectives, approaches, backgrounds, and/or abilities.

By sharing our process, we demonstrate that any field can (and should) be accessible to anyone with the right accommodations and modifications. We hope that this will motivate society to be open to new and different experiences and prompt the development of new tools and techniques that makes education more accessible. Because accessibility needs depend on the people and content involved, we also hope that this manuscript will encourage instructors to seek out and develop creative solutions to the accessibility issues that challenge their individual fields and styles of instruction, which could have profound benefits for both educators and students. Increasing the diversity of educators will also encourage students to pursue careers they may have never thought possible before as a result of seeing educators more like themselves ${ }^{45}$.

We still face challenges to make every aspect of the process as accessible as it can be, especially with the recent transition to virtual classes in the wake of COVID-19, and there is no doubt that there are more obstacles ahead. However, having experienced and observed first-hand the benefits for both Dr. Minkara and her students, we are driven to tackle them for the benefit of all. This experience has shown that creating an accessible environment and embracing diverse perspectives can have incredible benefits to education that extends beyond a single Bioengineering course.

\section{SUPPLEMENTARY INFORMATION}

The online version contains supplementary material available at https://doi.org/10.1007/s43683-021-000521.

\section{ACKNOWLEDGEMENTS}

MSM would like to acknowledge Andrew Leduc and Neda Kari Rezapour for their support as teaching assistants and for providing feedback on their experiences. MSM would also like to thank the Department of Bioengineering and Disability Services at Northeastern University for their support.

\section{REFERENCES}

${ }^{1}$ Bourne RRA, et al. Magnitude, temporal trends, and projections of the global prevalence of blindness and distance and near vision impairment: a systematic review and meta-analysis. Lancet Global Health 5(9):e888-e897, 2017.

${ }^{2}$ World Health Organization, Blindness and vision impairment. 2019 Accessed 25 March 2020; Available from: h ttps://www.who.int/news-room/fact-sheets/detail/blindnes s-and-visual-impairment.

${ }^{3}$ Erickson W, Lee C, von Schrader S. Disability statistics from the american community survey (ACS). 2017; Cornell University Yang-Tan Institute (YTI): Ithaca, NY.

${ }^{4}$ American Foundation for the Blind, Key Employment Statistics for People Who are Blind or Visually Impaired, in Research Navigator. 2017; American Foundation for the Blind.

${ }^{5} 2017$ American Community Survey. 2019; U.S. Census Bureau.

${ }^{6}$ Annual Report Fiscal Year 2018. 2018; American Printing House for the Blind, Inc.

${ }^{7}$ U.S. Department of Education, National Center for Education Statistics. Integrated Postsecondary Education Data System (IPEDS). 2018; Accessed 05 June 2020; Available from: https://nces.ed.gov/programs/coe/indicator_cha.as p\#info.

${ }^{8}$ Asebriy Z, Raghay S, Bencharef O. An assistive technology for braille users to support mathematical learning: a semantic retrieval system. Symmetry 10:547, 2018.

${ }^{9}$ Clark S, Baggaley J. Assistive software for disabled learners. Int Rev Res Open Distance Learn 5(3):1-6, 2004.

${ }^{10}$ Hersh M. Accessibility and usability of virtual learning environmemts, In: 2008 Eighth IEEE International Conference on Advanced Learning Technologies. 2008; 10381039. 
${ }^{11}$ Jones MG, Broadwell B. Visualization without vision: students with visual impairement, in visualization: theory and practice in science education. models and modeling in science education, Gilbert JK, Reiner M, Nakhleh, M (eds). 2008; Springer, Dordrecht.

${ }^{12}$ Jones MG, Taylor AR, Broadwell B. Concepts of scale held by students with visual impairment. $J$ Res Sci Teach 46(5):506-519, 2009.

${ }^{13}$ McDonald C, Rodrigues S. Sighted and visually impaired students' perspectives of illustrations, diagrams and drawings in school science. Wellcome Open Res 1:8, 2016.

${ }^{14}$ Melaku S, et al. Interlocking toy building blocks as handson learning modules for blind and visually impaired chemistry students. J Chem Educ 93(6):1049-1055, 2016.

${ }^{15}$ Minkara MS, et al. Implementation of protocols to enable doctoral training in physical and computational chemistry of a blind graduate student. J Chem Educ 92(8):1280-1283, 2015.

${ }^{16}$ Nazemi A, McMeekin DA, Murray I. Unbalanced chemical equations conversion to mark-up format and representation to vision impaired students. Comput Appl Eng Educ 23(6):805-812, 2015.

${ }^{17}$ Neely MB. Using technology and other assistive strategies to aid students with disabilities in performing chemisry lab tasks. J Chem Educ 84(10):1697-1701, 2007.

${ }^{18}$ Pereira F, et al. MOLinsight: a web portal for the processing of molecular structures by blind students. $J$ Chem Educ 88(3):361-362, 2011.

${ }^{19}$ Spuck T, et al. Research supporting multisensory engagement by BVI and sighted students to advance integrated learning of astronomy and computer science. In: 235th American Astronomical Society Meeting. 2020; Honolulu, HI.

${ }^{20}$ Stender AS, et al. Communicating science concepts to individuals with visual impairments using short learning modules. J Chem Educ 93(12):2052-2057, 2016.

${ }^{21}$ Supalo CA, et al. Low-cost laboratory adaptations for precollege students who are blind or visually impaired. $J$ Chem Educ 85(2):243-247, 2008.

${ }^{22}$ Eldridge C. National directory of blind teachers. $J$ Visual Impair Blind 78(3):123-128, 1984.

${ }^{23}$ Haworth DT, Eisch JJ. The professorial career of Clifford R. Haymaker: a life of chemistry imagined and bequeathed. $J$ Chem Educ 80(3):275, 2003.

${ }^{24}$ Minkara MS. Fellow blind scientists. Accessed 8 May 2020; Available from: https://monaminkara.com/fellow-blind-sci entists.

${ }^{25}$ American Association of Blind Teachers. Success. Available from: https://www.blindteachers.net/success.html.

${ }^{26}$ Coombs N. Making online teaching accessible: inclusive course design for students with disabilities. 1 edn. 2010; Jossey-Bass.
${ }^{27}$ Tobin TJ. Issues in preparing visually disabled instructors to teach online: a case study. Inform Technol Disabil E-J. 2002; 8(1).

${ }^{28}$ Minkara MS. Blind scientist tools. Accessed 28 April 2020. Available from: https://www.monaminkara.com/blind-scie ntist-tools/.

${ }^{29}$ King-Sears ME, Johnson TM, Berkeley S, Weiss MP, Peters-Burton EE, Evmenova AS, Menditto A, Hursh JC. An exploratory study of universal design for teaching chemistry to students with and without disabilities. Learn Disabil Quart 38(2):84-96, 2014.

${ }^{30}$ Meo G. Curriculum planning for all learners: applying universal design for learning (UDL) to a high school reading comprehension program. Prevent School Fail 52(2):21-30, 2008.

${ }^{31}$ Tobin TJ, Behling KT. Reach everyone, teach everyone: universal design for learning in higher education (Teaching and Learning in Higher Education). 1 edn. 2018; West Virginia University Press.

${ }^{32}$ Freeman S, Eddy SL, McDonough M, Smith MK, Okoroafor N, Jordt H, Wenderoth MP. Active learning boosts performance in STEM courses. Proc Nat Acad Sci 111(23):8410-8415, 2014.

${ }^{33}$ Prince M. Does active learning work? A review of the research. J Eng Educ 93(3):223-231, 2004.

${ }^{34}$ Felder RM, Brent R. Learning by doing. Chem Eng Educ 37(4):282-283, 2003.

${ }^{35}$ Felder RM, Brent R. Any questions? Chem Eng Educ 28(3):174-175, 1994.

${ }^{36}$ Heward WL. Want to improve the effectiveness of your lectures? Try guided notes. Acad Teach 1:33-46, 2004.

${ }^{37}$ Konrad M, Joseph LM, Eveleigh E. A meta-analytic review of guided notes. Educ Treat Child 32(3):421-444, 2009.

${ }^{38}$ Hartley J. Lecture handouts and student note-taking. Program Learn EducTechnol 13(2):58-64, 1976.

${ }^{39}$ Capp MJ. The effectiveness of universal design for learning: a meta-analysis of literature between 2013 and 2016. Int J Incl Educ 21(8):791-807, 2017.

${ }^{40}$ Steinfeld E, Maisel JL. Universal design: creating inclusive environments. 2012; Wiley.

${ }^{41}$ Center for Persons with Disabilities, U.S.U. Alternative Text. 2019; Available from: https://webaim.org/technique s/alttext/.

${ }^{42}$ Microsoft. Everything you need to know to write effective alt text. Available from: https://support.microsoft.com/enus/topic/everything-you-need-to-know-to-write-effective-al t-text-df98f884-ca3d-456c-807b-1a1fa82f5dc2.

${ }^{43}$ National Federation for the Blind, The Braille Literacy Crisis in America. 2009.

${ }^{44}$ HIMS. Polaris. Available from: https://hims-inc.com/prod uct/polaris/.

${ }^{45}$ Pritchard G. Disabled people as culturally relevant teachers. J Soc Incl 1(1):43-51, 2010. 